\title{
CNT nanosensors in the tumors treatment
}

\begin{abstract}
Magnetic fluid hyperthermia is the cancer treatment method based on heating of oncologic injured tissues together with covering them magnetic nanoparticles. It destroys selectively the tumor cells without damaging the health tissue. Thermal dose within a certain time causes tumor cell death that is divided by apoptosis and necrosis. Maximum safe temperatures deviate about $43^{\circ} \mathrm{C}$. They have to be safety measured at strong magnetic field impact. Then electric measurement methods fall firstly as interference unprotected and secondly as require electrical wires supplying to the irradiation zone. So, we study here the possibility of contactless Raman thermometer with help of nanotubes located nearby the tumors.
\end{abstract}

Keywords: CNT nanosensor, temperature, magnetic fluid hyperthermia, raman method
Volume 2 Issue 6 - 2017

\author{
Svyatoslav Yatsyshyn, Bohdan Stadnyk, \\ Rostyslav Samchenko \\ National University “Lviv Polytechnic”, Ukraine
}

\begin{abstract}
Correspondence: Svyatoslav Yatsyshyn, Institute of computer technologies, automatics and metrology, National University “Lviv Polytechnic", Ukraine, Tel +38068-5047368, Email slav.yat@gmail.com
\end{abstract}

Received: March 14, 2017| Published: July 05, 2017
Abbreviations: CNT, carbon nanotube; MNP, magnetic nanoparticle

\section{Introduction}

Magnetic fluid hyperthermia is the cancer treatment method based on heating of oncologic injured tissues using MNPs. Their controllable size allows bond and interact with biological entity once they are coated with biodegradable molecules such as dextran and others. Nowadays are widely researching composites based on hydrogels and MNPs since their properties are similar to those of living tissue. ${ }^{1}$ Heating MNPs together with hydrogels by altering magnetic field enables combining it with chemotherapy. Efficiency of treatment increases by 1.5-2.5 times. When cancer cells are heated over $42^{\circ} \mathrm{C}$ they begin to die, whereas healthy cells are unharmed till $48^{\circ} \mathrm{C} .{ }^{2}$ Based on numerous studies the maximum safe temperature has to be $\sim 43^{\circ} \mathrm{C} .{ }^{3}$ Temperature studies in micro volumes of tissues become the contemporary challenge since have be performed in-place.

\section{Discussion}

To apply hyperthermia, it becomes necessary the provision of desired temperature regime in volume being treated. Temperature measurement during hyperthermia is mostly invasive and provides results only at certain points. For safety the temperature field is previously examined by modeling only for body parts in which is planned to create overheating. It prevents formation of dangerous dots in tissue. For modeling the thermal processes, equations of heat transfer in biological systems are enjoyed. F.i., researchers apply rather simple Pennes' bioheat transfer equation ${ }^{4}$ that takes into account transfer between tissue and blood. MNPs key parameter in terms of heating by magnetic field is the released specific heat. Defining it as well as thermal conditions, it may be estimated the temperature field in body part being treated. Unfortunately, the gap arises between studies of tissue heating during its engineering testing and objects' measuring. Thus, the need to measure temperature directly in the field of irradiation arises. To exclude the impact of magnetic field on temperature, optical fiber thermometer may be applied. However then we cannot identify the controlled points.

While radiating the tissue by alternative field the very process of heating introduced MNPs complicates and becomes nonstationary. Another nonstationary process overlaps it; that is the process of heat removal from particles depending on temperature rise and MNPs concentration, on motion of particles of blood, lymph, and more. Preference should be given to direct temperature measurements of irradiated tissue, and it can be realized by nanosensors. Currently the established method for measuring the temperature and its jump at nanoscale is absent. We consider a number of possible methods of temperature measurement of biological objects, taking into account their noninvasiveness, minimization of methodic error and so on. Important is the last point. Our studies have demonstrated that minimum error while controlling small objects is guaranteed only when the ratio of volumetric and thermal characteristics of the nanosensor and the object is $10^{-3}$. To control the objects of size $10^{-3} \mathrm{~m}$, sensor's linear size should not exceed $10^{-4} \mathrm{~m}$. Not many temperature sensors meet such the requirements. Exactly they are considered below. For instance, liquid crystal films which change colors depending on temperature operate at $\mu$ m-thickness. ${ }^{5}$ Fluorescence polarization anisotropy of green protein is able to feel the temperature not harming the cell. ${ }^{6}$ Unfortunately this method refers to qualitative methods of thermometry.

Sensors with liquid- and solid-phase sensitive elements imply the availability of narrow tube with movable substance inside. Sensor's functioning roots in expanding the substance volume with temperature as it is in nanothermometer of $\mu \mathrm{m}$-length on basis of beta$\mathrm{Ga}_{2} \mathrm{O}_{3}$ nanotube of diameter 70-100 nm filled with $\mathrm{Au} / \mathrm{Si}$ alloy. ${ }^{6}$ For liquid thermometer exists the possibility of binding the changes of column length $\Delta h$ and the increase of temperature $\Delta T$. When thermo expanding factor is $10^{-4} 1 / \mathrm{K}$ and diameter of container for liquid $D=10 d$ ( $d$ is diameter of thermometric tube), we could obtain the equation of graduating characteristic: $\Delta h=0.067 d \Delta T$. Hereby, the sensitivity is reducing with diameter $d$. Similar equation is deduced also for micro- and nanosensors filled with solid-phase sensitive substance. Taking into account the behavior of liquid in capillary, equation of characteristic of nanothermometer with a liquid-phase sensitive element was defined: $\Delta h=C\left(T_{c}-6-T\right), K$. Here, $T_{c}$ depends on thermosensitive substance, constant $C$ of graduating characteristic comprises tube diameter $d$. Sensitivity of such nanosensor rises with decreasing $d$, whereas sensitivity of a nanosensor with a solid-phase sensitive element falls. ${ }^{7}$

In nanothermometry most universal is Raman method, apt for the direct temperature measurement of micro- and nano-objects $(0.1-100 \mu \mathrm{m})$ from cryogen till mid-high temperatures. It does not 
demand calibration. Determining the temperature here is based on the dependences of: a) ratio of intensities of Stokes and Anti-Stokes components of scattered radiation, b) the frequency shift of the object proper radiation. Our studies have envisaged that CNTs can serve as standard patterns. Their Raman spectrum is simple and well reproducible, and assures good reproducibility of received results. To decrease different components of error, $\mathrm{He}-\mathrm{Ne}$ laser of continuous effect and small power $1.2 \mathrm{~mW}$ as well as MS3501i spectrometer were used. It is necessary to underline that we need $50 \mathrm{mg}$ of CNTs packed into the plastic film to cover the square $1 \mathrm{~mm}^{2}$ of object chosen for study. $2^{\text {nd }}$ method is based on temperature determination due to the frequency shift of object radiation. Our studies envisage that shift is reducing from $1585.6 \mathrm{~cm}^{-1}$ at $15^{\circ} \mathrm{C}$ to $1576.1 \mathrm{~cm}^{-1}$ at $250^{\circ} \mathrm{C}$. Temperature factor of frequency change is $-0.041 \mathrm{~cm}^{-1} /{ }^{\circ} \mathrm{C}$. Gained result at $14.12^{\circ} \mathrm{C}$ was estimated with expanded error $0.58 \%$ and combined standard uncertainty $0.3 \%$ at the credence level 0.95 , and expanded coefficient 1.96. Method was elaborated; just a number of modes for reducing twice the uncertainty were studied. They included the modes of defining shift by: a) maximal value of the peak intensity; b) averaging the mean integral value of anti-Stokes component on the basis of perfecting the hardware and software. ${ }^{?}$

\section{Conclusion}

Challenge in medicine is to develop current and/or create new treatment methods, including measurement techniques and standards, which rely on nanoscaled materials. Within range $270-330 \mathrm{~K}$ for Raman method, as one of few direct methods of thermometry, the sensitive carbon nanotubes are treated to be universal calibration artifacts for measuring nanosized objects at tumors' magnetic hyperthermia process.

\section{Acknowledgements}

None.

\section{Conflict of interest}

The author declares no conflict of interest.

\section{References}

1. Tabatabaei SN, Lapointe J, Martel S. Magnetic nanoparticles encapsulated in hydrogel as hyperthermic actuators for microrobots designed to operate in the vascular network. IEEE; 2009. p. 1-6.

2. Blue L. Fluid dynamics and heating of magnetic nanoparticles in simulated blood vessels; 2009.

3. Khanafer K, Vafai K. Synthesis of mathematical models representing bioheat transfer. Adv in num heat transfer. 2009;3:1-28.

4. Zablotskii V, Lunov O, Gomez Polo C. Magnetic heating by tunable arrays of nanoparticles in cancer therapy. Acta Phys Pol. 2009;115(1):413-417.

5. A precise nanothermometer for intracellular temperature mapping. Nanowerk Spotlight; 2012.

6. Gong $\mathrm{N}$, Wang $\mathrm{CY}$, Chen $\mathrm{Y}$, et al. $\mathrm{Au}(\mathrm{Si})$-filled $\mathrm{b}-\mathrm{Ga}_{2} \mathrm{O}_{3}$ nanotubes as wide range high temperature nanothermometers. Appl Phys Let. 2008;92(7):073101.

7. Yatsyshyn S, Stadnyk B, Lutsyk Ya, et al. Handbook of thermometry and nanothermometry. Spain: IFSA Publishing; 2015. 